\title{
The telestroke and thrombolysis therapy in diabetic stroke patients
}

\author{
Thomas I. Nathaniel ${ }^{*}$, Chibueze Ubah, Leah Wormack and Jordan Gainey
}

\begin{abstract}
Objective: Several controversial findings have been reported on treatment outcomes for diabetic stroke patients that received thrombolysis therapy in the hospital. We determined whether the association between telestroke technology, thrombolysis therapy and clinical risk factors in diabetic acute ischemic stroke may result in the inclusion or exclusion or more diabetic ischemic stroke patients for thrombolysis therapy.

Methods: Retrospective data that comprises of a total of 3202 acute ischemic stroke patients from a regional stroke registry that contained telestroke and non telestroke patients with a primary diagnosis of acute ischemic stroke of which 312 were identified as diabetic stroke patients were used in this study. Multivariate logistic regression models were used to determine the associated pre-clinical risk factors, and demographics associated with recombinant tissue plasminogen activator (rtPA) therapy in a subset of diabetic acute ischemic stroke patients in the telestroke and nontelestroke settings.

Results: In the telestroke, only higher International Normalized Ratio (INR) [odds ratio, OR $=0.063(0.003-1.347,95 \%$ confidence interval (Cl)] was associated with exclusion from thrombolysis. Direct admission [OR, 3.141 (1-9.867), 95\% $\mathrm{Cl}$ and telestroke [OR, $4.87(1.834-12.928), 95 \% \mathrm{Cl}$ ] were independent predictors in the inclusion for thrombolysis therapy. In the non telestroke, older age (> 80 years) [(OR), $0.955(0.922-0.989), 95 \% \mathrm{Cl}]$, higher blood glucose level [OR, 0.994 (0.99-0.999); 95\% Cl], higher INR [OR, $0.113(0.014-0.944) ; 95 \%$ Cl], and renal insufficiency [OR, 0.163 (0.033-0.791); 95\% CI] were associated with exclusion while higher NIH stroke scale [OR, 1.068 (1.009-1.13); 95\% Cl] was associated with inclusion for thrombolysis in the non telestroke.
\end{abstract}

Conclusion: The non-telestroke setting admitted more diabetic stroke patients to the hospital, but more were excluded from thrombolysis therapy when compared with the telestroke setting. Measures to improve clinical risk factors that excluded more diabetic ischemic stroke patients in the non telestroke will improve the use of thrombolysis in the treatment of diabetic acute ischemic stroke patients.

Keywords: Acute stroke, Diabetes mellitus, Exclusion, Inclusion, Telestroke, Non telestroke

\section{Introduction}

Diabetes mellitus is a frequently identified comorbid risk factor in acute ischemic stroke. The risk of ischemic stroke in diabetic patients is twofold higher when compared to people without diabetes [1]. This underlies the close relationship between these two co-occurring common diseases. Though the disease processes are closely related, controversial findings have been reported on

*Correspondence: nathanit@greenvillemed.sc.ed

School of Medicine-Greenville, University of South Carolina, Greenville, SC 29605, USA treatment outcomes for diabetic stroke patients that received thrombolysis therapy [2-6]. This is because the management of diabetic stroke patients is complicated, and this results in most of the observed controversial outcomes.

Although diabetes is not an absolute or relative exclusion criteria for thrombolysis, a low rate of thrombolysis therapy has been reported in diabetic ischemic stroke patients due to concerns over poorer outcomes [7]. Proposed factors for the poor response include stroke severity [8], a higher risk of developing post stroke hyperglycemia [9] and vascular risk factors [2]. Thrombolysis 
is known to produce better outcomes in stroke patients when compared with diabetic stroke patients [6], and clinical trials [10] did not suggest the withholding of thrombolysis therapy from diabetic stroke patients [11]. Moreover, existing studies suggest that the lower rate of thrombolysis therapy in diabetic stroke patients does not appear to be related to contraindications for thrombolysis because a comparison of contraindications for thrombolysis between ischemic stroke patients with and without diabetes did not reveal a significant difference [12].

It has been shown that a practice-based model of telestroke can manage pretreatment clinical risk factors for thrombolysis therapy relaxing the criteria for the inclusion or exclusion for thrombolysis in ischemic stroke patients [13]. Although the telestroke is known with favorable outcomes in acute ischemic stroke [13-17], however, the effect of telestroke technology in enhancing the use of thrombolysis therapy in diabetic stroke patients when compared with treatment is not known. We know that several studies in non telestroke settings, reveal controversial findings on treatment outcomes for diabetic stroke patients that received thrombolysis therapy. While some studies report poorer outcomes in diabetic ischemic stroke patients when compared with non-diabetic acute ischemic stroke patients [2-4], others have shown the safety and beneficial effects of recombinant tissue plasminogen activator (rtPA) $[5,6]$. It is also known that treatment outcomes in telestroke programs have been favorable, and consistent with good expectations in several studies in acute ischemic stroke [13-19]. What is not known is whether the association between telestroke technology, thrombolysis therapy and clinical risk factors in diabetic acute ischemic stroke may result in the inclusion or exclusion of diabetic ischemic stroke patients for thrombolysis therapy. We investigated this issue in a population of diabetic acute ischemic stroke patients treated in a telestroke and compared our findings with a non telestroke setting. We used multivariate models to predict the odds of including more diabetic stroke patients for thrombolysis therapy in the telestroke when compared with the non telestroke setting. The current study investigated telestroke technology in the use of thrombolysis therapy in diabetic acute ischemic stroke patients with various baseline clinical risk factors.

\section{Method}

\section{Patient selection and baseline characteristics}

Retrospective data were collected from the acute ischemic stroke registry of Greenville Health System (GHS) between January 2010 and June 2016. The registry has been described in our previous studies [20-23]. Patients were selected with prospective inclusion of consecutive patients with diabetic acute ischemic stroke treated in a stroke center (non-telestroke) and telestroke network. Data for the various pre-clinical risk factors was extracted including; atrial fib/flutter, carotid artery stenosis, congestive heart failure, depression, dyslipidemia, coronary artery disease, family history of stroke, hormone replacement therapy, hypertension, migraine, obesity, peripheral vascular disease, previous stroke, previous TIA, prosthetic heart valve, renal insufficiency, sleep apnea, smoking, substance abuse. Additional variables from time of admission were also included. The National Institutes of Health stroke scale (NIHSS) was used to evaluate severity of neurologic impairment. Laboratory analysis for the concentrations of total cholesterol, low-density lipoprotein cholesterol (LDL), triglycerides, lipids, high-density lipoprotein cholesterol (HDL), blood glucose and creatine were obtained at admission. Values for systolic blood pressure, diastolic blood pressure and International Normalized Ratio (INR) were determined.

Upon admission, all patients underwent brain computed tomography. Patients with subarachnoid and intracerebral hemorrhage were excluded in our analysis. A standardized stroke protocol was used in all patients, including T2-weighted, T1-weighted, and diffusionweighted images. Data on symptom onset time and the admission to Emergency Department (ED) for both telestroke and non telestroke diabetic stroke patients were collected. Patients that were directly admitted to the ED or with emergency medical services (EMS) and those with indirect admission by being transferred to the ED in the telestroke or non telestroke from another hospital were also identified. Data on patient demographics, including age, sex, race, and ethnicity were also extracted Information on the ambulation status prior to event, during and at discharge were also collected. Ethical approval was obtained from the Institutional Review Board of Greenville Health System and the institutional Committee for Ethics.

\section{Data analysis}

The SPSS package version 20 (Chicago, IL, USA) for Windows was used for all statistical analyses and $\mathrm{P}<0.05$ was used to establish statistical significance for all comparisons between groups. We used univariate analysis to analyze baseline characteristics including age, gender, medical history, prestroke treatments and admission parameters such as blood glucose and stroke severity. This allowed us to determine baseline or pre-clinical risk factors that were associated with inclusion or exclusion for recombinant tissue plasminogen activator (rtPA). All discrete variables were represented as number (percentage) and comparisons between groups were made using 
Pearson's Chi Squared analyses. Descriptive statistics were calculated for the demographic and clinical characteristics of patients. All continuous variables were presented as mean (STD), and comparisons between groups were determined using the Student's $T$ test. All variables presented in Tables 1 and 2 were analyzed using univariate analysis while multivariate models were used to identify significant associations with exclusion or inclusion for thrombolysis therapy in the whole diabetic stroke population in telestroke and non telestroke settings (see Tables 3, 4 and 5). Adjustments in the multivariate analysis were based on univariate significance. Subsequent multivariate logistic regression was based on risk factors in diabetic stroke patients associated with thrombolysis therapy and specific for telestroke or non telestroke identified by the univariate analysis. This analysis identified independent predictors of exclusion or inclusion for thrombolysis therapy. The multivariable model was built by stepwise conditional logistic regression. We used a backward procedure as a follow-up to test the modeling strategy, while the test for the log likelihood was used to assess the suitability of fit and to compare nested models. All variables that produced changes $>10 \%$ of the odds ratio (OR) when eliminated were considered to be confounding variables, while variables with a value of $\mathrm{P}<0.01$ on univariate testing were included. All stepwise regression models were assessed using Hosmer \& Lemeshow test, Cox \& Snell $\mathrm{R}^{2}$ and Classification Plots. Multicollinearity of variables were assessed with variance inflation factor analysis to confirm independence of variables included in regression model.

\section{Results}

A total of 3202 acute ischemic stroke patients were collected from the stroke registry, 312 were identified as diabetic stroke patients. Of the 312,180 were in the nontelestroke setting and 132 in the telestroke setting. Comparisons between the baseline demographic and clinical characteristics of telestroke and non-telestroke diabetic acute ischemic stroke patients are presented in Table 1. Telestroke patients tended to be younger $(65.9 \pm 12.3 \mathrm{vs}$. $69.3 \pm 12.7)$, have a higher body mass index $(32.2 \pm 7.5$ vs. $29.5 \pm 7.3$ ), less likely to have a history of atrial fibrillation $(8.3 \%$ vs. $21.7 \%)$, or a previous stroke $(23.5 \%$ vs. $40.6 \%$ ), more likely to have a family history of stroke $(18.2 \%$ vs. $7.8 \%)$ and obese ( $64.4 \%$ vs. $43.3 \%)$. At the time of presentation, telestroke patients had a lower creatinine (1.2 \pm 1.0 vs. $1.5 \pm 1.1)$ and lower diastolic blood pressure (78.8 \pm 17.1 vs. $81.8 \pm 19.2$ ). Telestroke patients tended to have a better ambulatory status at baseline, at the time of presentation and at discharge. Telestroke patients were more likely to be directly admitted ( $71.2 \%$ vs. $11.7 \%)$ and more likely to receive rtPA ( $86.4 \%$ vs. $37.8 \%)$. Multivariate analysis reveals three factors more associated with telestroke patients than non-telestroke patients: obesity [OR, 2.493 (1.135-5.475); 95\% CI, $\mathrm{P}=0.023]$, direct admission [OR, 14.248 (6.012-33.766); 95\% CI, $\mathrm{P}<0.001$ ], and rtPA administration obesity [OR, 1.068 (1.009-1.13); 95\% CI, $\mathrm{P}<0.001]$.

As shown in Table 2, non-telestroke patients who received rtPA were more likely to be younger $(66.8 \pm 13.5$ vs. $70.8 \pm 12)$, have a lower blood glucose level $(171 \pm 88$ vs. $210.9 \pm 126.8)$, have a lower creatinine $1.3 \pm 0.7$ vs. $1.6 \pm 1.3)$, a lower INR $(1.1 \pm 0.1$ vs. $1.2 \pm 0.4)$, and present a better ambulatory status at baseline than patients who did not receive rtPA. In the telestroke, patients who received rtPA were more likely to have a worse ambulatory status at presentation and more likely to be directly admitted (77.2\% vs. 33.3\%). Multivariate analysis reveals four factors associated with rtPA (Table 3). Higher INR [OR, 0.139 (0.029-0.67); 95\% CI, $\mathrm{P}=0.014]$ and congestive heart failure [OR, 0.329 (0.124-0.878); 95\% $\mathrm{CI}, \mathrm{P}=0.026]$ were associated with rtPA exclusion while direct admission [OR $=3.141$ (1-9.867); 95\% $\mathrm{CI}, \mathrm{P}=0.050]$ and being a telestroke patient [OR, 4.87 (1.834-12.928); 95\% CI, $\mathrm{P}=0.0001$ ] were more associated with rtPA inclusion. The ROC curve for the predictive power of the regression model is presented in Fig. 1. The discriminating capability of the model was very good as shown by the ROC curve, with area under the curve (AUROC) of AUROC $=0.774$ (95\% CI, 0.712-0.836, $\mathrm{P}<0.00$ ). In the non-telestroke (Table 4$)$, older age (> 80 years) [OR, 0.955 (0.922$0.989 ; 95 \% \mathrm{CI}, \mathrm{P}=0.009$ ], higher blood glucose level [OR, 0.994 (0.99-0.999);95\% CI, P=0.0014], higher INR [OR, $0.113(0.014-0.944) ; 95 \% \mathrm{CI}, \mathrm{P}=0.004]$, and renal insufficiency [OR, $0.163(0.033-0.024) ; 95 \% \mathrm{CI}$, $\mathrm{P}=0.004]$, were all associated with rtPA exclusion while higher NIH stroke scale [OR, 1.068 (1.0091.13);95\% $\mathrm{CI}, \mathrm{P}=0.023$ ] was associated with $\mathrm{rtPA}$ inclusion. As presented in Fig. 2, the predictive power of the logistic regression was strong. The area under the curve (AUROC) is 0.678 (95\% CI, 0.639-0.718, $\mathrm{P}<0.01$ ). In the telestroke (Table 5), only higher INR [OR, 0.063 (0.003-1.347) 95\% CI, P=0.077]) was associated with rtPA exclusion and the association was not significant. The predictive model power of the logistic regression was strong (Fig. 3), AUROC $=0.678$ (95\% CI, 0.639-0.718, $\mathrm{P}<0.05$ ).

\section{Discussion}

In a diabetic acute ischemic stroke population, patients that present with obesity, directly admitted to emergency department, and received thrombolysis therapy have higher odds of being associated with the telestroke 
Table 1 Demographic factors and clinical characteristics of acute ischemic stroke patients with a history of diabetes divided by telestroke status

\begin{tabular}{|c|c|c|c|}
\hline Characteristic & $\begin{array}{l}\text { Non-telestroke } \\
(\mathrm{N}=180)\end{array}$ & $\begin{array}{l}\text { Telestroke } \\
(\mathrm{N}=132)\end{array}$ & P-value \\
\hline \multicolumn{4}{|l|}{ Patient age in years } \\
\hline Mean \pm SD & $69.3 \pm 12.7$ & $65.9 \pm 12.3$ & $0.020^{*}$ \\
\hline \multicolumn{4}{|l|}{ Age group: no. (\%) } \\
\hline$<50$ years & $14(7.8)$ & $11(8.3)$ & 0.069 \\
\hline $50-59$ & $26(14.4)$ & $20(15.2)$ & \\
\hline $60-69$ & $43(23.9)$ & $49(37.1)$ & \\
\hline $70-79$ & $54(30)$ & $33(25)$ & \\
\hline$\geq 80$ & $43(23.9)$ & $19(14.4)$ & \\
\hline \multicolumn{4}{|l|}{ Gender: no. (\%) } \\
\hline Male & $88(48.9)$ & $69(52.3)$ & 0.555 \\
\hline Female & $92(51.1)$ & $63(47.7)$ & \\
\hline \multicolumn{4}{|l|}{ Race: no. (\%) } \\
\hline Caucasian & $127(70.6)$ & $102(77.3)$ & 0.212 \\
\hline African-American & $32(17.8)$ & $19(14.4)$ & \\
\hline Other & $3(1.7)$ & $3(2.3)$ & \\
\hline Hispanic ethnicity: no. (\%) & $5(2.8)$ & $6(4.5)$ & 0.403 \\
\hline \multicolumn{4}{|l|}{ Body mass index } \\
\hline Mean $\pm S D$ & $29.5 \pm 7.3$ & $32.2 \pm 7.5$ & $0.001^{*}$ \\
\hline \multicolumn{4}{|l|}{ Medical history: no. (\%) } \\
\hline Atrial fib/flutter & $39(21.7)$ & $11(8.3)$ & $0.002^{*}$ \\
\hline Carotid artery stenosis & $12(6.7)$ & $6(4.5)$ & 0.427 \\
\hline Congestive heart failure & $28(15.6)$ & $17(12.9)$ & 0.506 \\
\hline Coronary artery disease & $80(44.4)$ & $62(47)$ & 0.658 \\
\hline Depression & $1(0.6)$ & $27(20.5)$ & $<0.001^{*}$ \\
\hline Dyslipidemia & $124(68.9)$ & $92(69.7)$ & 0.879 \\
\hline Family history of stroke & $14(7.8)$ & $24(18.2)$ & $0.006^{*}$ \\
\hline $\begin{array}{l}\text { Hormone replacement } \\
\text { therapy }\end{array}$ & $3(1.7)$ & $3(2.3)$ & 0.7 \\
\hline Hypertension & $165(91.7)$ & $123(93.2)$ & 0.62 \\
\hline Migraine & $5(2.8)$ & $4(3)$ & 0.895 \\
\hline Obesity & $78(43.3)$ & $85(64.4)$ & $<0.001^{*}$ \\
\hline Peripheral vascular disease & $23(12.8)$ & $11(8.3)$ & 0.213 \\
\hline Previous stroke & $73(40.6)$ & $31(23.5)$ & $0.002^{*}$ \\
\hline Previous TIA & $22(12.2)$ & $9(6.8)$ & 0.115 \\
\hline Prosthetic heart valve & $1(0.6)$ & $0(0)$ & 0.391 \\
\hline Renal insufficiency & $24(13.3)$ & $9(6.8)$ & 0.065 \\
\hline Sleep apnea & $0(0)$ & $11(8.3)$ & $<0.001^{*}$ \\
\hline Smoking & $41(22.8)$ & $25(18.9)$ & 0.412 \\
\hline Substance abuse & $5(2.8)$ & $2(1.5)$ & 0.457 \\
\hline \multicolumn{4}{|l|}{ Initial NIH stroke scale } \\
\hline Mean \pm SD & $10.8 \pm 8.6$ & $8.9 \pm 7.6$ & 0.063 \\
\hline \multicolumn{4}{|l|}{ Initial labs and vitals } \\
\hline Total cholesterol & $165.7 \pm 56.9$ & $165.1 \pm 43.4$ & 0.885 \\
\hline Triglycerides & $157.3 \pm 118.4$ & $159.3 \pm 106.1$ & 0.307 \\
\hline $\mathrm{HDL}$ & $39.4 \pm 12.9$ & $37.8 \pm 11.7$ & 0.565 \\
\hline LDL & $95.8 \pm 36.5$ & $98.4 \pm 35.3$ & 0.889 \\
\hline Lipids & $7.6 \pm 2.1$ & $7.6 \pm 2.1$ & 0.067 \\
\hline
\end{tabular}

Table 1 (continued)

\begin{tabular}{|c|c|c|c|}
\hline Characteristic & $\begin{array}{l}\text { Non-telestroke } \\
(\mathrm{N}=180)\end{array}$ & $\begin{array}{l}\text { Telestroke } \\
(\mathrm{N}=132)\end{array}$ & P-value \\
\hline Blood glucose & $195.8 \pm 115.2$ & $173 \pm 97$ & 0.054 \\
\hline Creatinine & $1.5 \pm 1.1$ & $1.2 \pm 1$ & $0.007^{*}$ \\
\hline INR & $1.1 \pm 0.3$ & $1 \pm 0.2$ & 0.074 \\
\hline Heart rate & $84.1 \pm 19.2$ & $80.4 \pm 16.9$ & 0.097 \\
\hline Systolic blood pressure & $155.9 \pm 33.2$ & $150.6 \pm 23.6$ & 0.158 \\
\hline Diastolic blood pressure & $81.8 \pm 19.2$ & $78.8 \pm 17.1$ & $<0.001^{*}$ \\
\hline \multicolumn{4}{|c|}{ Medications prior to admission: no. (\%) } \\
\hline $\begin{array}{l}\text { Antiplatelet or anticoagu- } \\
\text { lant }\end{array}$ & $112(62.2)$ & $79(59.8)$ & 0.671 \\
\hline Antihypertensive & $150(83.3)$ & $114(86.4)$ & 0.464 \\
\hline Cholesterol reducer & $113(62.8)$ & $91(68.9)$ & 0.258 \\
\hline Diabetic medication & $128(71.1)$ & $100(75.8)$ & 0.361 \\
\hline \multicolumn{4}{|c|}{ Ambulation status prior to event: no. (\%) } \\
\hline Ambulate independently & $148(82.2)$ & $121(91.7)$ & 0.106 \\
\hline Ambulate with assistance & $12(6.7)$ & $3(2.3)$ & \\
\hline Unable to ambulate & $11(6.1)$ & $5(3.8)$ & \\
\hline Not documented & $9(5)$ & $3(2.3)$ & \\
\hline \multicolumn{4}{|c|}{ Ambulation status on admission: no. (\%) } \\
\hline Ambulate independently & $20(11.1)$ & $26(19.7)$ & $0.016^{*}$ \\
\hline Ambulate with assistance & $18(10)$ & $23(17.4)$ & \\
\hline Unable to ambulate & $75(41.7)$ & $39(29.5)$ & \\
\hline Not documented & $67(37.2)$ & $44(33.3)$ & \\
\hline \multicolumn{4}{|c|}{ Ambulation status on discharge: no. (\%) } \\
\hline Ambulate independently & $69(38.3)$ & $61(46.2)$ & $0.044^{*}$ \\
\hline Ambulate with assistance & $46(25.6)$ & $42(31.8)$ & \\
\hline Unable to ambulate & $47(26.1)$ & $18(13.6)$ & \\
\hline Not documented & $18(10)$ & $11(8.3)$ & \\
\hline \multicolumn{4}{|l|}{ First care received: no. (\%) } \\
\hline Emergency department & $159(88.3)$ & $38(28.8)$ & $<0.001^{*}$ \\
\hline Direct admission & $21(11.7)$ & $94(71.2)$ & \\
\hline rtPA administration & $68(37.8)$ & $114(86.4)$ & $<0.001^{*}$ \\
\hline Improved ambulation & 109 (60.6) & $89(67.4)$ & 0.213 \\
\hline \multicolumn{4}{|c|}{$\begin{array}{l}\text { Continuous variables are represented as Mean } \pm \text { S.D. and comparisons between } \\
\text { groups are made with a Student's T Test. Discrete variables are represented as } \\
\text { Count (Percent Frequency) and comparisons between groups were made using } \\
\text { Pearson's Chi Squared }\end{array}$} \\
\hline${ }^{*} \mathrm{P}<0.05$ & & & \\
\hline
\end{tabular}

setting. Following the adjustment for comorbidities, the telestroke setting represents the strongest predictor for the administration of thrombolysis therapy. In both telestroke and non-telestroke diabetic acute ischemic stroke patients, direct admission represents a predictor for administration of thrombolysis therapy. In the univariate analysis, non-telestroke diabetic stroke patients who received thrombolysis were more likely to be younger, have a lower blood glucose level, lower creatinine, lower INR, and present with a better ambulatory status at baseline than the patients 
Table 2 Clinical characteristics, medical history, and presenting symptoms of acute ischemic stroke patients with a history of diabetes stratified by rtPA status and telestroke status

\begin{tabular}{|c|c|c|c|c|c|c|}
\hline \multirow[t]{2}{*}{ Characteristic } & \multicolumn{3}{|c|}{ Non-telestroke } & \multicolumn{3}{|l|}{ Telestroke } \\
\hline & $\begin{array}{l}\text { No rtPA } \\
(\mathrm{N}=112)\end{array}$ & $\begin{array}{l}\text { rtPA } \\
(\mathrm{N}=68)\end{array}$ & P-value & $\begin{array}{l}\text { No rtPA } \\
(\mathrm{N}=18)\end{array}$ & $\begin{array}{l}\text { rtPA } \\
(\mathrm{N}=114)\end{array}$ & P-value \\
\hline \multicolumn{7}{|l|}{ Patient age in years } \\
\hline Mean \pm SD & $70.8 \pm 12$ & $66.8 \pm 13.5$ & $0.043^{*}$ & $66.9 \pm 13.2$ & $65.8 \pm 12.2$ & 0.727 \\
\hline \multicolumn{7}{|l|}{ Age group: no. (\%) } \\
\hline$<50$ years & $6(5.4)$ & $8(11.8)$ & 0.014 & $1(5.6)$ & $10(8.8)$ & 0.362 \\
\hline $50-59$ & $16(14.3)$ & $10(14.7)$ & & $5(27.8)$ & $15(13.2)$ & \\
\hline $60-69$ & $20(17.9)$ & $23(33.8)$ & & $5(27.8)$ & $44(38.6)$ & \\
\hline $70-79$ & $42(37.5)$ & $12(17.6)$ & & $3(16.7)$ & $30(26.3)$ & \\
\hline$\geq 80$ & $28(25)$ & $15(22.1)$ & & $4(22.2)$ & $15(13.2)$ & \\
\hline \multicolumn{7}{|l|}{ Gender: no. (\%) } \\
\hline Male & $49(43.8)$ & $39(57.4)$ & 0.077 & $9(50)$ & $60(52.6)$ & 0.835 \\
\hline Female & $63(56.3)$ & $29(42.6)$ & & $9(50)$ & $54(47.4)$ & \\
\hline Race: no. (\%) & & & & (0) & $(0)$ & \\
\hline Caucasian & $73(65.2)$ & $54(79.4)$ & 0.6 & $14(77.8)$ & $88(77.2)$ & 0.74 \\
\hline African-American & $21(18.8)$ & $11(16.2)$ & & $2(11.1)$ & $17(14.9)$ & \\
\hline Other & $3(2.7)$ & $0(0)$ & & $0(0)$ & $3(2.6)$ & \\
\hline Hispanic ethnicity: no. (\%) & $2(1.8)$ & $3(4.4)$ & 0.917 & $0(0)$ & $6(5.3)$ & 0.319 \\
\hline \multicolumn{7}{|l|}{ Body mass index } \\
\hline Mean \pm SD & $29.6 \pm 7.6$ & $29.1 \pm 7$ & 0.657 & $31 \pm 7$ & $32.4 \pm 7.6$ & 0.447 \\
\hline \multicolumn{7}{|l|}{ Medical history: no. (\%) } \\
\hline Atrial fib/flutter & $30(26.8)$ & $9(13.2)$ & 0.032 & $2(11.1)$ & $9(7.9)$ & 0.646 \\
\hline Carotid artery stenosis & $10(8.9)$ & $2(2.9)$ & 0.118 & $0(0)$ & $6(5.3)$ & 0.319 \\
\hline Congestive heart failure & $21(18.8)$ & $7(10.3)$ & 0.129 & $4(22.2)$ & $13(11.4)$ & 0.203 \\
\hline Coronary artery disease & $48(42.9)$ & $32(47.1)$ & 0.582 & $9(50)$ & $53(46.5)$ & 0.782 \\
\hline Depression & $1(0.9)$ & $0(0)$ & 0.435 & $3(16.7)$ & $24(21.1)$ & 0.668 \\
\hline Dyslipidemia & $76(67.9)$ & $48(70.6)$ & 0.701 & $13(72.2)$ & $79(69.3)$ & 0.802 \\
\hline Family history of stroke & $8(7.1)$ & $6(8.8)$ & 0.683 & $0(0)$ & $24(21.1)$ & 0.031 \\
\hline Hormone replacement therapy & $2(1.8)$ & $1(1.5)$ & 0.873 & $0(0)$ & $3(2.6)$ & 0.486 \\
\hline Hypertension & $103(92)$ & $62(91.2)$ & 0.853 & $16(88.9)$ & $107(93.9)$ & 0.437 \\
\hline Migraine & $1(0.9)$ & $4(5.9)$ & 0.048 & $1(5.6)$ & $3(2.6)$ & 0.501 \\
\hline Obesity & $48(42.9)$ & $30(44.1)$ & 0.869 & $12(66.7)$ & $73(64)$ & 0.828 \\
\hline Peripheral vascular disease & $17(15.2)$ & $6(8.8)$ & 0.216 & $1(5.6)$ & $10(8.8)$ & 0.646 \\
\hline Previous stroke & $50(44.6)$ & $23(33.8)$ & 0.152 & $5(27.8)$ & $26(22.8)$ & 0.644 \\
\hline Previous TIA & $13(11.6)$ & $9(13.2)$ & 0.746 & $2(11.1)$ & $7(6.1)$ & 0.437 \\
\hline Prosthetic heart valve & $1(0.9)$ & $0(0)$ & 0.435 & (0) & $(0)$ & \\
\hline Renal insufficiency & $20(17.9)$ & $4(5.9)$ & 0.022 & $1(5.6)$ & $8(7)$ & 0.819 \\
\hline Smoking & $23(20.5)$ & $18(26.5)$ & 0.357 & $4(22.2)$ & $21(18.4)$ & 0.702 \\
\hline Substance abuse & $2(1.8)$ & $3(4.4)$ & 0.299 & $0(0)$ & $2(1.8)$ & 0.571 \\
\hline \multicolumn{7}{|l|}{ Initial NIH stroke scale } \\
\hline Mean \pm SD & $10.3 \pm 9.2$ & $11.3 \pm 7.8$ & 0.462 & $10.7 \pm 9.4$ & $8.6 \pm 7.4$ & 0.34 \\
\hline \multicolumn{7}{|l|}{ Initial labs and vitals } \\
\hline Total cholesterol & $168.1 \pm 62.8$ & $162.4 \pm 48.4$ & 0.547 & $160.9 \pm 53$ & $165.7 \pm 42$ & 0.682 \\
\hline Triglycerides & $157.6 \pm 125.1$ & $157 \pm 109.7$ & 0.976 & $128.4 \pm 68.7$ & $163.9 \pm 110.1$ & 0.213 \\
\hline $\mathrm{HDL}$ & $40.2 \pm 14.1$ & $38.3 \pm 11$ & 0.379 & $38.4 \pm 13.5$ & $37.8 \pm 11.5$ & 0.843 \\
\hline LDL & $95.2 \pm 34.3$ & $96.6 \pm 39.4$ & 0.816 & $98.9 \pm 50.6$ & $98.3 \pm 32.7$ & 0.945 \\
\hline Lipids & $7.8 \pm 2.2$ & $7.3 \pm 1.9$ & 0.171 & $7.6 \pm 2.4$ & $7.6 \pm 2.1$ & 0.945 \\
\hline Blood glucose & $210.9 \pm 126.8$ & $171 \pm 88.3$ & $0.014^{*}$ & $184.2 \pm 133.1$ & $171.3 \pm 90.6$ & 0.602 \\
\hline
\end{tabular}


Table 2 (continued)

\begin{tabular}{|c|c|c|c|c|c|c|}
\hline \multirow[t]{2}{*}{ Characteristic } & \multicolumn{3}{|c|}{ Non-telestroke } & \multicolumn{3}{|l|}{ Telestroke } \\
\hline & $\begin{array}{l}\text { No rtPA } \\
(\mathrm{N}=112)\end{array}$ & $\begin{array}{l}\text { rtPA } \\
(\mathrm{N}=68)\end{array}$ & P-value & $\begin{array}{l}\text { No rtPA } \\
(\mathrm{N}=18)\end{array}$ & $\begin{array}{l}\text { rtPA } \\
(\mathrm{N}=114)\end{array}$ & P-value \\
\hline Creatinine & $1.6 \pm 1.3$ & $1.3 \pm 0.7$ & $0.032^{*}$ & $1.6 \pm 2.3$ & $1.2 \pm 0.6$ & 0.375 \\
\hline INR & $1.2 \pm 0.4$ & $1.1 \pm 0.1$ & $0.002^{*}$ & $1.2 \pm 0.5$ & $1 \pm 0.1$ & 0.347 \\
\hline Heart rate & $85.5 \pm 21.2$ & $81.8 \pm 15.2$ & 0.216 & $77.9 \pm 15.4$ & $80.8 \pm 17.1$ & 0.505 \\
\hline Systolic blood pressure & $155.1 \pm 35$ & $157.3 \pm 30.1$ & 0.669 & $151.7 \pm 22$ & $150.4 \pm 24$ & 0.835 \\
\hline Diastolic blood pressure & $82.5 \pm 20.6$ & $80.7 \pm 16.8$ & 0.555 & $77.8 \pm 14.4$ & $79 \pm 17.5$ & 0.779 \\
\hline \multicolumn{7}{|l|}{ Medications prior to admission: no. (\%) } \\
\hline Antiplatelet or anticoagulant & $72(64.3)$ & $40(58.8)$ & 0.464 & $12(66.7)$ & $67(58.8)$ & 0.525 \\
\hline Antihypertensive & $93(83)$ & $57(83.8)$ & 0.891 & $14(77.8)$ & $100(87.7)$ & 0.253 \\
\hline Cholesterol reducer & $70(62.5)$ & $43(63.2)$ & 0.921 & $15(83.3)$ & $76(66.7)$ & 0.156 \\
\hline Diabetic medication & 78 (69.6) & $50(73.5)$ & 0.577 & $12(66.7)$ & $88(77.2)$ & 0.333 \\
\hline Ambulation status prior to event: no. (\%) & & & & (0) & $(0)$ & \\
\hline Ambulate independently & 85 (75.9) & $63(92.6)$ & $0.028^{*}$ & $15(83.3)$ & $106(93)$ & 0.511 \\
\hline Ambulate with assistance & $11(9.8)$ & $1(1.5)$ & & $1(5.6)$ & $2(1.8)$ & \\
\hline Unable to ambulate & $8(7.1)$ & $3(4.4)$ & & (0) & $4(3.5)$ & \\
\hline Not documented & $8(7.1)$ & $1(1.5)$ & & (0) & $2(1.8)$ & \\
\hline Ambulation status on admission: no. (\%) & & & & (0) & $(0)$ & \\
\hline Ambulate independently & $16(14.3)$ & $4(5.9)$ & 0.107 & $6(33.3)$ & $20(17.5)$ & $0.016^{*}$ \\
\hline Ambulate with assistance & $14(12.5)$ & $4(5.9)$ & & $0(0)$ & $23(20.2)$ & \\
\hline Unable to ambulate & $45(40.2)$ & $30(44.1)$ & & $9(50)$ & $30(26.3)$ & \\
\hline Not documented & $37(33)$ & $30(44.1)$ & & $3(16.7)$ & $41(36)$ & \\
\hline \multicolumn{7}{|l|}{ Ambulation status on discharge: no. (\%) } \\
\hline Ambulate independently & $39(34.8)$ & $30(44.1)$ & 0.223 & $9(50)$ & $52(45.6)$ & 0.328 \\
\hline Ambulate With assistance & $29(25.9)$ & $17(25)$ & & $3(16.7)$ & $39(34.2)$ & \\
\hline Unable to ambulate & $29(25.9)$ & $18(26.5)$ & & $3(16.7)$ & $15(13.2)$ & \\
\hline Not documented & $15(13.4)$ & $3(4.4)$ & & $3(16.7)$ & $8(7)$ & \\
\hline \multicolumn{7}{|l|}{ First care received: no. (\%) } \\
\hline Emergency department & $100(89.3)$ & 59 (86.8) & 0.609 & $12(66.7)$ & $26(22.8)$ & $<0.001^{*}$ \\
\hline Direct admission & $12(10.7)$ & $9(13.2)$ & & $6(33.3)$ & $88(77.2)$ & \\
\hline Improved ambulation & $63(56.3)$ & $46(67.6)$ & 0.129 & $14(77.8)$ & $75(65.8)$ & 0.313 \\
\hline
\end{tabular}

who did not receive thrombolysis. In the telestroke setting, diabetic acute ischemic stroke patients who received thrombolysis were more likely to have a worse ambulatory status at presentation and more likely to be directly admitted to the emergency department.

In the adjusted analysis for the total diabetic stroke population, only direct admission and being treated in the telestroke setting were independent variables associated with administration of thrombolysis therapy. The non-telestroke setting admitted more diabetic stroke patients, but more were excluded from thrombolysis therapy when compared with the telestroke setting. This may be connected with a higher rate of hospital admission of patients with highly variable clinical risk factors, resulting in the exclusion of more admitted patients from thrombolysis therapy when compared with the telestroke setting. In the adjusted analysis for the non-telestroke setting, age $(>80)$, higher blood glucose level, and renal insufficiency were all associated with exclusion from thrombolysis. The benefits of thrombolysis therapy have been shown in many studies [20, 2230]. Findings indicate higher functional dependency in stroke patients older than 80 years that received thrombolysis therapy [31-36]. The observed poor functional outcome appeared to be linked to poorer baseline clinical conditions such as congestive heart failure, ischemic heart disease, and hypertension 
Table 3 A stepwise regression model to elucidate clinical factors more associated rtPA inclusion in the total study population of diabetic acute ischemic stroke patients

\begin{tabular}{lrlrl}
\hline & B value & Adj. odds ratio & Wald & P value \\
\hline INR & -1.971 & $0.139(0.029-0.67)$ & 6.054 & $0.014^{*}$ \\
$\begin{array}{l}\text { Congestive heart } \\
\quad-1.111\end{array}$ & $0.329(0.124-0.878)$ & 4.930 & $0.026^{*}$ \\
$\quad$ Direct admission & 1.145 & $3.141(1-9.867)$ & 3.842 & 0.050 \\
Telestroke & 1.583 & $4.87(1.834-12.928)$ & 10.097 & $0.001^{*}$ \\
Constant & 2.256 & 9.541 & 6.418 & $0.011^{*}$ \\
\hline
\end{tabular}

Positive $B$ values ( $\mathrm{ddj}, \mathrm{OR}>1$ ) denote variables more associated with rtPA inclusion while negative $B$ values $(A d j . O R<1)$ denote variables more associated with rtPA exclusion. Multicollinearity and interactions among independent variables were checked. Hosmer-Lemeshow test $(P=0.084)$, Cox \& Snell $\left(R^{2}=0.260\right)$, classification table (overall correctly classified percentage $=74.3 \%$ ) were applied to check the model fitness

${ }^{*} \mathrm{P}<0.05$

Table 4 A stepwise regression model to elucidate clinical factors more associated rtPA inclusion in the nontelestroke population

\begin{tabular}{lrlrl}
\hline & B value & Adj. odds ratio & Wald & P value \\
\hline Higher age & -0.046 & $0.955(0.922-0.989)$ & 6.797 & $0.009^{*}$ \\
NIH stroke scale & 0.066 & $1.068(1.009-1.13)$ & 5.190 & $0.023^{*}$ \\
Blood glucose level & -0.006 & $0.994(0.99-0.999)$ & 6.037 & $0.014^{*}$ \\
INR & -2.180 & $0.113(0.014-0.944)$ & 4.054 & $0.044^{*}$ \\
Renal insufficiency & -1.817 & $0.163(0.033-0.791)$ & 5.064 & $0.024^{*}$ \\
Constant & 6.225 & 505.460 & 11.330 & $0.001^{*}$ \\
\hline
\end{tabular}

Positive $B$ values ( $A d j, O R>1$ ) denote variables more associated with rtPA inclusion while negative $B$ values ( $A d j . O R<1$ ) denote variables more associated with rtPA exclusion. Multicollinearity and interactions among independent variables were checked. Hosmer-Lemeshow test $(P=0.493)$, Cox \& Snell $\left(R^{2}=0.224\right)$, classification table (overall correctly classified percentage $\left.=70.8 \%\right)$ were applied to check the model fitness

${ }^{*} \mathrm{P}<0.05$

Table 5 A stepwise regression model to elucidate clinical factors more associated rtPA inclusion in the telestroke population

\begin{tabular}{lrlll}
\hline & B value & Adj. odds ratio & Wald & P value \\
\hline INR & -2.758 & $0.063(0.003-1.347)$ & 3.130 & 0.077 \\
Constant & 5.155 & 173.322 & 8.724 & $0.003^{*}$ \\
\hline
\end{tabular}

Positive $B$ values (Adj, OR $>1$ ) denote variables more associated with rtPA inclusion while negative $B$ values $(A d j . O R<1$ ) denote variables more associated with rtPA exclusion. Multicollinearity and interactions among independent variables were checked. Cox \& Snell $\left(R^{2}=0.051\right)$, and a classification table (overall correctly classified percentage $=91.0 \%$ ) were applied to check the model fitness

${ }^{*} \mathrm{P}<0.05$

in older stroke patients. In the current study, our results indicate that pre-stroke functional status, higher blood glucose level, age older than 80, and

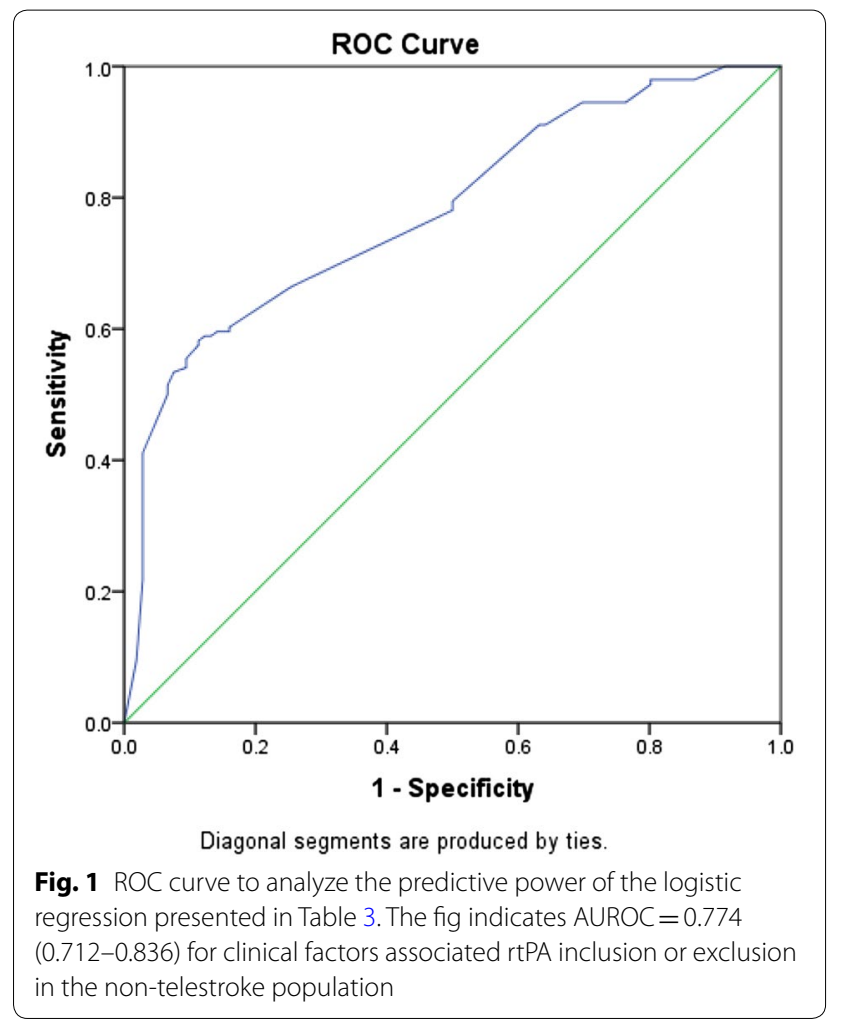

renal insufficiency were all associated with exclusion from thrombolysis therapy in diabetic stroke patients treated in the non-telestroke setting. These factors

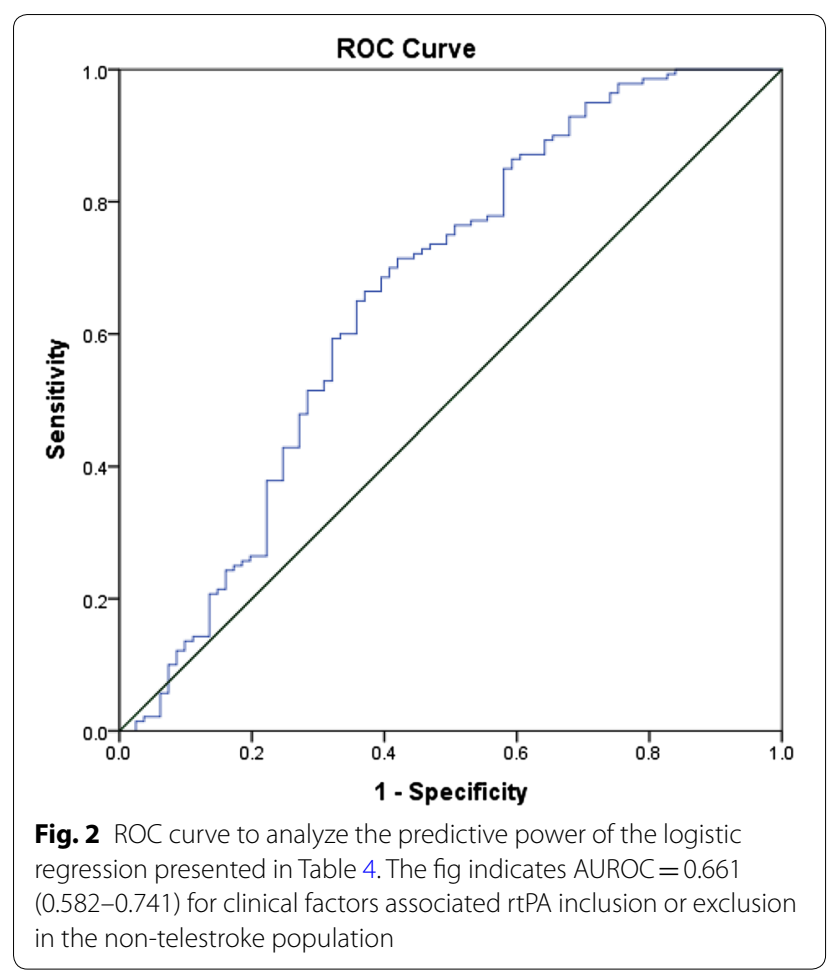




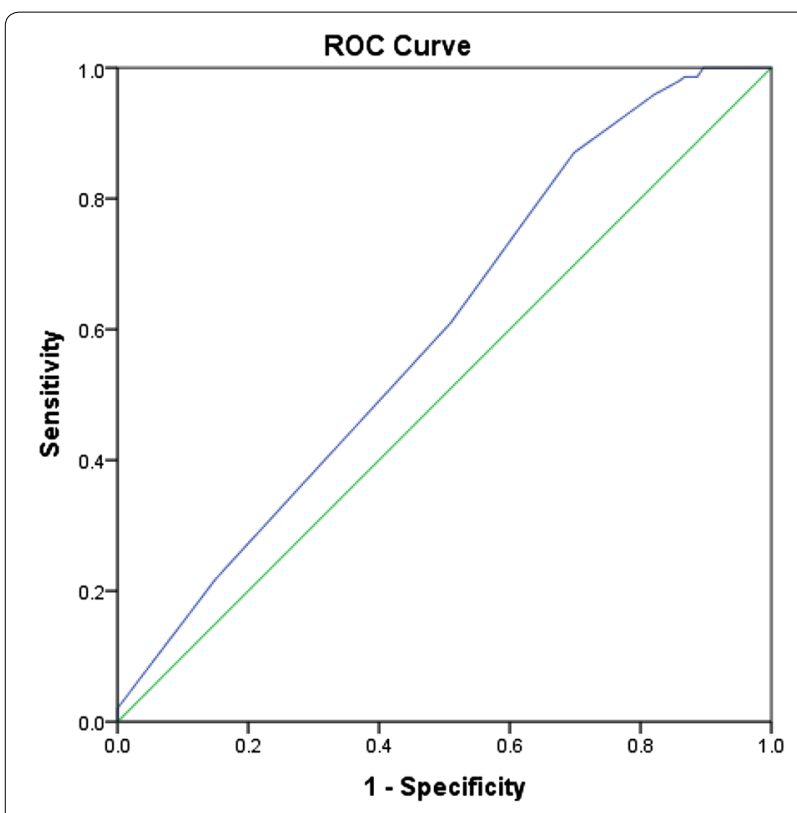

Diagonal segments are produced by ties.

Fig. 3 ROC curve to analyze the predictive power of the logistic regression presented in Table 5. The fig indicates $A U R O C=0.678$ (0.639-0.718) for clinical factors associated rtPA inclusion or exclusion in the non-telestroke population

have been shown to influence functional outcome in longitudinal studies among elderly stroke patients $[20,37,38]$. Stroke-related mortality is linked to age as a major independent risk factor mainly because elderly acute ischemic patients are more susceptible to complications and have more comorbidities than their younger counterparts [39]. However, advanced age should not be a contraindication for thrombolysis in diabetic stroke patients. Instead, the course of treatment should be decided on a case-by-case basis after a detailed evaluation of existing comorbidities and pre-stroke clinical risks as well as the potential benefits of thrombolytic therapy for each individual old diabetic acute ischemic stroke patient.

A major finding in this study is that our multivariate model predicted a direct association of treatment in the telestroke setting as an independent variable with the highest odds for the inclusion of diabetic stroke patients for thrombolysis therapy. Moreover, following adjustment for baseline demographic and clinical risk factors in the telestroke network, only diabetic stroke patients with higher INR were excluded from thrombolysis, and the effect was not significant. These findings differ from the non-telestroke setting in which diabetic stroke patients with increased age, higher blood glucose level, higher INR, and renal insufficiency were all pre-clinical risk factors that predicted exclusion from thrombolysis therapy. The finding that in the non-telestroke setting, diabetic stroke patients with complicated pre-clinical risk factors were associated with a higher likelihood of exclusion from thrombolysis therapy, suggests a more stringent exclusion criteria when compared with the telestroke setting. Therefore, it is possible that telestroke technology provides a real-world clinical setting that streamlines in-hospital evaluation with less stringent exclusion criteria, allowing stroke neurologist to consult quickly on whether or not administer thrombolysis therapy. This may enable an increase in the rate of use and efficiency of the timeline for administration of thrombolysis in the treatment of diabetic acute ischemic stroke patients.

There are limitations to our study. First, our study is limited by its retrospective design, although data was collected using an established prospective stroke registry, a risk of selection bias is possible. Furthermore, this is unicenter stroke registry and does not allow for the generalization of our findings. Moreover, information about the management of diabetes mellitus (type I or type II) was not included in our analysis. The relatively small groups of patients of diabetic stroke patients did not increase the power of our analysis. The strengths of our study are that in the non-telestroke setting, increased age, higher blood glucose level, renal insufficiency were pre-clinical risk factors that predicted the exclusion from thrombolysis therapy, while only INR predicted a non-significant exclusion from thrombolysis therapy in the telestroke setting. Our multivariate model was able to identify treatment in the telestroke setting as an independent variable with the highest prediction for the inclusion of diabetic stroke patients for thrombolysis therapy. Finally, we found that in older diabetic stroke patients ( $>80$ years), exclusion maybe linked with pre-treatment functional status that includes history of higher blood glucose level, higher INR, and renal insufficiency.

\section{Conclusion}

Diabetes is not an exclusion criterion for thrombolysis, however, a low rate of thrombolysis therapy has been reported in diabetic acute ischemic stroke patients. More studies are necessary to determine how identified exclusion risk factors in the non-telestroke setting can be improved to provide a real-world clinical setting with less stringent exclusion criteria for thrombolysis therapy.

\section{Abbreviations}

rtPA: recombinant tissue plasminogen activator; TIA: trans ischemic attack; OR: adjusted odd ratio; GWTG: get with the guideline; AHA: American Heart Association; NIH scores: National Institute of Health scores; AUROC: area under 
the curve; ROC: receiver operator characteristic; $\mathrm{Cl}$ : confidence interval; CAD: coronary artery disease; MAP: mean arterial pressure; CHF: congestive heart failure; PVD: peripheral vascular disease; GHS: Greenville Health System.

\section{Authors' contributions}

TIN and JG designed the concept, experimental design, data analysis, while CU and LW critically revised the drafts last version of this manuscript. All authors read and approved the final manuscript.

\section{Acknowledgements}

We thank the stroke unit of Greenville Health system for helping in the data collection.

\section{Competing interests}

The authors declare that they have no competing interests.

\section{Availability of data and materials}

All materials are available for use from the corresponding author.

\section{Consent for publication}

Not applicable.

\section{Ethics approval and consent to participate}

This study was performed with the approval of the Institutional Review Board of Greenville Health System and the institutional Committee for Ethics. Being a retrospective data analysis with blinded data, no consent was needed.

\section{Funding}

This study was funded by the Fullerton Foundation (Grant No. 78029867).

\section{Publisher's Note}

Springer Nature remains neutral with regard to jurisdictional claims in published maps and institutional affiliations.

Received: 4 February 2019 Accepted: 2 March 2019

Published online: 09 May 2019

\section{References}

1. Tun NN, Arunagirinathan G, Munshi SK, Pappachan JM. Diabetes mellitus and stroke: a clinical update. World J Diabetes. 2017;8(6):235-48.

2. Braun KF, Otter W, Sandor SM, Standl E, Schnell O. All-cause in-hospital mortality and comorbidity in diabetic and non-diabetic patients with stroke. Diabetes Res Clin Pract. 2012:98(1):164-8.

3. MacDougal EL, Herman WH, Wing JJ, Morgenstern LB, Lisabeth LD. Diabetes and ischaemic stroke outcome. Diabet Med. 2018;35(9):1249-57.

4. Mapoure YN, Ba H, Ayeah CM, Kenmegne C, Luma HN, Njamnshi AK Acute stroke patients with newly diagnosed diabetes mellitus have poorer outcomes than those with previously diagnosed diabetes mellitus. J Stroke Cerebrovasc Dis. 2018;27(9):2327-35.

5. Fuentes B, Martinez-Sanchez P, de Lecinana MA, et al. Diabetes and previous stroke: hazards for intravenous thrombolysis? Eur J Neurol. 2012;19(4):587-93.

6. Mishra NK, Ahmed N, Davalos A, et al. Thrombolysis outcomes in acute ischemic stroke patients with prior stroke and diabetes mellitus. Neurology. 2011;77(21):1866-72.

7. Reeves MJ, Vaidya RS, Fonarow GC, et al. Quality of care and outcomes in patients with diabetes hospitalized with ischemic stroke findings from get with the guidelines-stroke. Stroke. 2010;41(5):E409-17.

8. Kamouchi M, Matsuki T, Hata J, et al. Prestroke glycemic control is associated with the functional outcome in acute ischemic stroke the Fukuoka Stroke Registry. Stroke. 2011;42(10):2788-94.

9. Gray CS, Scott JF, French JM, Alberti K, O'Connell JE. Prevalence and prediction of unrecognised diabetes mellitus and impaired glucose tolerance following acute stroke. Age Ageing. 2004;33(1):71-7.

10. Kwiatkowski T, Libman R, Tilley BC, et al. The impact of imbalances in baseline stroke severity on outcome in the National Institute of
Neurological Disorders and Stroke recombinant tissue plasminogen activator stroke study. Ann Emerg Med. 2005;45(4):377-84.

11. Generalized efficacy of t-PA for acute stroke. Subgroup analysis of the NINDS t-PA stroke trial. Stroke. 1997;28(1):209.

12. Fuentes B, Cruz-Herranz A, Martinez-Sanchez P, et al. Acute ischemic stroke patients with diabetes should not be excluded from intravenous thrombolysis. J Thromb Thrombolysis. 2014;38(4):522-7.

13. Al Kasab S, Adams RJ, Debenham E, Jones DJ, Holmstedt CA. Medical University of South Carolina Telestroke: a telemedicine facilitated network for stroke treatment in South Carolina a progress report. Telemed E-Health. 2017;23(8):674-7.

14. Bagot $\mathrm{KL}$, Bladin CF, Vu M, et al. Exploring the benefits of a stroke telemedicine programme: an organisational and societal perspective. J Telemed Telecare. 2016;22(8):489-94.

15. Itrat A, Taqui A, Cerejo R, et al. Telemedicine in prehospital stroke evaluation and thrombolysis taking stroke treatment to the doorstep. JAMA Neurol. 2016;73(2):162-8.

16. Kulcsar M, Gilchrist S, George MG. Improving stroke outcomes in rural areas through telestroke programs: an examination of barriers, facilitators, and state policies. Telemed E-Health. 2014;20(1):3-10.

17. LaMonte MP, Bahouth MN, Xiao Y, Hu P, Baquet CR, Mackenzie CF. Outcomes from a comprehensive stroke telemedicine program. Telemed J E-Health. 2008;14(4):339-44

18. Gonzalez MA, Hanna N, Rodrigo ME, Satler LF, Waksman R. Reliability of prehospital real-time cellular video phone in assessing the simplified National Institutes of Health Stroke Scale in patients with acute stroke a novel telemedicine technology. Stroke. 2011;42(6):1522-7.

19. Demaerschalk BM, Raman R, Ernstrom K, Meyer BC. Efficacy of telemedicine for stroke: pooled analysis of the Stroke Team Remote Evaluation Using a Digital Observation Camera (STRokE DOC) and STRokE DOC arizona telestroke trials. Telemed E-Health. 2012;18(3):230-7.

20. Nathaniel TI, Gainey J, Blum B, Montgomery C, Ervin L, Madeline L. Clinical risk factors in thrombolysis therapy: telestroke versus nontelestroke. J Stroke Cerebrovasc Dis. 2018;27(9):2524-33.

21. Nathaniel TI, Cochran T, Chaves J, et al. Co-morbid conditions in use of recombinant tissue plasminogen activator (rt-PA) for the treatment of acute ischaemic stroke. Brain Inj. 2016;30(10):1261-5.

22. Gainey J, Brecthtel J, Blum B, et al. Functional outcome measures of recombinant tissue plasminogen activator-treated stroke patients in the telestroke technology. J Exp Neurosci. 2018;12:1-11.

23. Wapshott T, Blum B, Williams K, Nathaniel TI. Investigation of gender differences and exclusive criteria in a diabetic acute ischemic stroke population treated with recombinant tissue-type plasminogen activator (rtPA). J Vasc Interv Neurol. 2017;9(6):26-32.

24. Barral M, Lassalle L, Dargazanli C, et al. Predictors of favorable outcome after mechanical thrombectomy for anterior circulation acute ischemic stroke in octogenarians. J Neuroradiol. 2018;45(4):211-6.

25. Fazzone $B$, Morris $G$, Black $L A$, et al. Exclusion and inclusion criteria for thrombolytic therapy in an ischemic stroke population.e 4(2): 1112. J Neurol Disord Strok. 2016;4(2):1-5.

26. Cochran T, Chaves J, Fulmer E, et al. Analysis of comorbidities in ischemic stroke patient. Neurology. 2014;82(10):278.

27. Blum B, Wormack L, Holtel M, et al. Gender and thrombolysis therapy in stroke patients with incidence of dyslipidemia. BMC Womens Health. 2019;19:11.

28. Gainey J, Blum B, Bowie B, et al. Stroke and dyslipidemia: clinical risk factors in the telestroke versus non-telestroke. Lipids Health Dis. 2018;17:226.

29. Brecthel L, Gainey J, Penwell A, Nathaniel TI. Predictors of thrombolysis in the telestroke and non telestroke settings for hypertensive acute ischemic stroke patients. BMC Neurol. 2018;18:215.

30. Colello MJ, Ivey LE, Gainey J, et al. Pharmacological thrombolysis for acute ischemic stroke treatment: gender differences in clinical risk factors. Adv Med Sci. 2018;63(1):100-6.

31. Sandercock P, Wardlaw JM, Lindley RI. The benefits and harms of intravenous thrombolysis with recombinant tissue plasminogen activator within $6 \mathrm{~h}$ of acute ischemic stroke (the third international stroke trial [IST-3]); a randomised controlled trial Lancet. Lancet. 2012;379:2352-63.

32. Yayan J. Effectiveness of alteplase in the very elderly after acute ischemic stroke. Clin Interv Aging. 2013;8:963-74. 
33. Sagnier S, Galli P, Poli M, et al. The impact of intravenous thrombolysis on outcome of patients with acute ischemic stroke after 90 years old. BMC Geriatr. 2016;16:156.

34. Fredwall M, Sternberg S, Blackhurst D, Lee A, Leacock R, Nathaniel TI. Gender differences in exclusion criteria for recombinant tissue-type plasminogen activator. J Stroke Cerebrovasc Dis. 2016;25(11):2569-74.

35. Gainey J, Brecthtel L, Blum B, et al. Functional outcome measures of recombinant tissue plasminogen activator-treated stroke patients in the telestroke technology. J Exp Neurosci. 2018;12:1179069518793412.
36. Lawson TR, Brown IE, Westerkam DL, et al. Tissue plasminogen activator (rt-PA) in acute ischemic stroke: outcomes associated with ambulation. Restor Neurol Neurosci. 2015;33(3):301-8.

37. Demaerschalk BM. Scientific rationale for the inclusion and exclusion criteria for intravenous alteplase in acute ischemic stroke: a statement for healthcare professionals from the American Heart Association/American Stroke Association. Stroke. 2016;47(11):581.

38. Fugate J, Rabinstein A. Absolute and relative contraindications to IV rt-PA for acute ischemic stroke. Neurohospitalist. 2015:5(3):110-21.

39. Fu GR, Yuan WQ, Du WL, et al. Risk factors associated with recurrent strokes in young and elderly patients: a hospital-based study. Int J Gerontol. 2015;9(2):63-6.
Ready to submit your research? Choose BMC and benefit from:

- fast, convenient online submission

- thorough peer review by experienced researchers in your field

- rapid publication on acceptance

- support for research data, including large and complex data types

- gold Open Access which fosters wider collaboration and increased citations

- maximum visibility for your research: over $100 \mathrm{M}$ website views per year

At BMC, research is always in progress.

Learn more biomedcentral.com/submissions 